\title{
Trennung und Bestimmung von 17-Ketosteroiden aus dem Harn durch Dünnschichtchromatographie
}

\author{
Von V. Graef und HJ. Staudinger \\ Aus dem Physiologisch-Chemischen Institut der Universität Gießen (Direktor: Prof. Dr. Hj. Staudinger)
}

(Eingegangen am 15. April 1967)

Es wird über cine Routinemethode zur Trennung und Bestimmung der sieben wichtigsten 17-Ketosteroide im menschlichen Harn berichtet. Der Harnextrakt wird nach enzymatischer Hydrolyse und Solvolyse der Steroidkonjugate in zwei verschiedenen Lösungsmittelsystemen durch Dünnschichtchromatographic nach dem Durchlaufverfahren getrennt. Auf diese Weise wird cine gute Trennung sowohl der 11-Desoxy- wie auch der 11-oxygenierten 17-Ketosteroide erzielt. Die Zuvcrlässigkeit der Methode wurde durch Wiederfindungsversuche und Mehrfachbestimmungen geprüft.

$\Lambda$ routine method is reported for the separation and determination of the seven main 17-ketosteroids in human urine. The urine extract is separated by thin-layer chromatography with the overrun technique in two different solvent systems after enzymatic hydrolysis and solvolysis of the stcroid conjugates. By this method a good separation of the 11-desoxy-and the 11-oxygenated 17-ket osteroids was obtained. 'The reliability of the method was cstablished by recovery experiments and by duplicate assays.

Seit Entwicklung der Dünnschichtchromatographie sind verschiedene Methoden (1-6) zur Trennung der 17Ketosteroide aus dem Harn beschrieben worden. Die Trennung der sieben wichtigsten im menschlichen Harn vorkommenden 17-Ketosteroide (Dehydroepiandrosteron, Androsteron, Ätiocholanolon, $11 \beta$-Hydroxyandrosteron, 11-Ketoandrosteron, $11 \beta$-Hydroxyätiocholanolon und 11-Ketoätiocholanolon) ist nicht ohne Schwierigkeiten möglich, da sich diese sieben Substanzen auf einer üblichen Chromatographieplatte von $20 \times 20 \mathrm{~cm}$, d. h. auf einer Laufstrecke von etwa $18 \mathrm{~cm}$, nicht genügend trennen lassen. Bei einer zweidimensionalen Dünnschichtchromatographie (4) ist es schwierig, die Flecken zu lokalisieren, wenn man die Platte nicht besprühen will, um die Flecken sichtbar zu machen. Das erschwert aber eine quantitative Bestimmung. Eine optimale Trennung der sieben Steroide erreichen wir mit der „Durchlaufchromatographie“. Zur Trennung und Isolierung der 11-Desoxy-17-ketosteroide einerseits und der 11-oxygenierten 17-Ketosteroide andererseits wird der Harnextrakt auf zwei verschiedenen Dünnschichtplatten in zwei verschiedenen Systemen chromatographiert. Zur Bestimmung der 11-oxygenierten Steroide, die in geringerer Menge ausgeschieden werden, wird eine größere Menge Harnextrakt verwendet.

\section{Methodik \\ Reagenzien}

Åthanol (aldchydfrci): $1 /$ Äthanol (abs.) wird mit $3 \mathrm{~g}$ Natriumborhydrid an einer Füllkörperkolonne destillicrt.

Methanol: p. a. (Fa. Ricdcl-de Hac̈n) wird über eine Füllkörperkolonnc destillicrt.

Äthylacetat: wird mit ${ }^{1 /}{ }_{10}$ Vol. 8proz. NatriumhydrogencarbonatLösung und drcimal mit Wasser ausgeschüttelt, über Calciumchlorid getrocknct und destillicrt.

Chloroform: p. a. (Fa. Merck).

Acctatpuffer pH 5,2:32,4 g wasscrfreies Natriumacetat und 7,15 ml Eiscssig werden mit dest. Wasser auf $250 \mathrm{~m} /$ aufgefüllt.

$\beta$-Glucuronidasc/Aryl-Sulfatase aus Helix pomatia (Fa. C. F. Boehringer, Mannheim).

Aluminiumoxid: neutral, $\Lambda$ kt. II (Gebr. Giulini, Ludwigshafen). Aluminiumoxid G: für dic Dünnschichtchromatographie (Fa. Merck).

m-Dinitrobenzol (1proz. in aldehydfreiem Alkohol): Die Lösung muß im Dunkeln aufbewahrt werden und soll nicht älter als eine Woche scin.
Zimmermann-Reagenz: zum Besprühen der Dünnschichtplatten. 2proz. äthanol. m-Dinitrobenzol-Lösung und methanol. Kaliiauge (7,5 g Kaliumhydroxid in $50 \mathrm{~m} /$ Methanol) werden vor Gebrauch im Verhältnis 2:1 gemischt.

Dehydrocpiandrosteron, Androsteron und Ätiocholanolon (Fa. Vister, Casatenovo Brianza (Como)).

$11 \beta$-Hydroxyandrosteron, 11-Ketoandrosteron, 11 $\beta$-Hydroxyätiocholanolon und 11-Ketoätiocholanolon (Fa. Ikapharm Ltd., Ramat-Gan, Israel).

Hydrolyse (nach Baulieu und JAYLE) (7).

$20 \mathrm{~m} /$ zentrifugierter Harn werden mit Eisessig auf $\mathrm{pH} 5,2$ eingestellt, mit $50 \mu l \beta$-Glucuronidase/Aryl-Sulfatase und $1 \mathrm{ml}$ Acctatpuffer pH 5,2 versetzt und $24 \mathrm{Stdn}$. bei $37^{\circ}$ inkubiert. Dann säucrt man mit 50proz. Schwefelsäure auf pH 1 an, löst im Harn $4 \mathrm{~g}$ Natriumchlorid und extrahiert dreimal mit je $50 \mathrm{ml}$ Äthylacetat. Die vercinigten Extrakte werden $24 \mathrm{Stdn}$. bei $37^{\circ}$ aufbewahrt und nach dem Abkühlen zweimal mit je $20 \mathrm{~m} l \mathrm{~N} \mathrm{NaOH}$ und zweimal mit je $20 \mathrm{ml}$ dest. Wasser ausgeschüttelt. Nach Trocknen über wasserfreiem Natriumsulfat dampft man die Lösung im Vakuum ein.

Wenn der Harnextrakt stark gefärbt ist, wird seine Lösung in $3 \mathrm{~m} l$ Chloroform durch eine kleine Säule aus Aluminiumoxid (Akt. II) $(1 \times 3 \mathrm{~cm})$ filtriert und dreimal mit je $3 \mathrm{~m} /$ und einmal mit $5 \mathrm{~m} /$ Chloroform nachgespült. Das Eluat dampft man im Vakuum ein und nimmt den Rückstand in $10 \mathrm{ml}$ Methanol auf. Zur Dünnschichtchromatographie dampft man $1 \mathrm{~m} l$ der Lösung (=2 $\mathrm{m} l$ Harn, zur Bestimmung von Dehydroepiandrosteron, Androsteron und Ätiocholanolon) und $3 \mathrm{~m} l$ der Lösung ( $=6 \mathrm{~m} / \mathrm{Harn}$, zur Bestimmung der 11-oxygenierten Steroide) in spitzen Zentrifugenröhrchen mit Schliff am Rotationsverdampfer im Vakuum ein.

\section{Diinuschjicbtcluromatograpljie}

Man benötigt Dünnschichtplatten von verschiedener Aktivität. Zur Bestimmung von Dehydroepiandrosteron, Androsteron und Ätiocholanolon werden Platten von geringer Aktivität verwendet. Glasplatten $(20 \times 20 \mathrm{~cm})$ werden mit einem Streichgerät mit einer $250 \mu$ dicken Schicht Aluminiumoxid $G$ bestrichen, indem man $30 \mathrm{~g}$ Adsorbens und $50 \mathrm{~m} /$ dest. Wasser etwa 20 Sek. kräftig schüttelt und mit dieser Suspension 5 Glasplatten bestreicht. Die Chromatographieplatten werden 2 Stdn. bei $125^{\circ}$ getrocknet. Dann läßt man sie auf Raumtemperatur abkühlen und bewahrt sie $10 \mathrm{Stdn}$. bei $20^{\circ}$ in cinem Exsikkator auf, auf dessen Boden sich ein angefeuchtetes Stück Zellstoff $(10 \times 10 \mathrm{~cm})$ befindet. Danach entfernt man den Zellstoff und bewahrt die Platten im Exsikkator ohne Trockenmittel auf, wo sie einen konstanten Feuchtigkeitsgehalt behalten. - Die stark aktivierten Platten zur Bestimmung von $11 \beta$-Hydroxyandrosteron, 11-Ketoandrosteron, $11 \beta$-Hydroxyätiocholanolon und 11-Ketoätiocholanolon werden 4 Stdn. bei $125^{\circ}$ erhitzt. Dann schaltet man den Trockenschrank ab und läßt die Platten über Nacht im Schrank abkühlen. Sie werden im Exsikkator über Blaugel aufbewahrt. 
Für dic Durchlaufchromatographie legt man auf den Boden einer handclsüblichen Chromatographickammer 6 Glasscheiben $(20 \times 5 \times$ $0,4 \mathrm{~cm}$ ) und kleidet die Kammer mit Filtrierpapier aus. Wenn man jetzt eine Dünnschichtplatte senkrecht in die Kammer stellt, soll die Platte etwa $1,2 \mathrm{~cm}$ aus der Kammer herausragen. Die Chromatographickammer wird mit so viel Laufmittel gefüllt, daß dic Flüssigkeit etwa $0,5 \mathrm{~cm}$ über den Glasplatten am Boden steht. Zur Chromatographie deckt man die Kammer mit zwei Glasplatten $28 \times 8 \times$ $0,25 \mathrm{~cm} z u, z w i s c h e n$ denen man einen Spalt offen läßt, durch den die Dünnschichtplatte senkrecht in die Kammer gestellt wird. Man schiebt beide Glasplatten mit sanftem Druck zusammen und gegen die Dünnschichtplatte und verschließt die noch vorhandenen Offnungen zu beiden Seiten der Chromatographicplatte mit Objektträgern, an deren Unterseite man $3 \mathrm{~mm}$ breite Kunststoffstreifen festgeklebt hat. Auf diese Weise ist dic Kammer fest verschlossen. Sobald die Lösungsmittelfront das oberc Ende der Dünnschichtplatte, das aus der Kammer herausragt, erreicht hat, kann das Laufmittel verdampfen.

Zur Bestimmung von Debydroepiandrosteron, Androsteron und Ätiocbolanolon wird der Verdampfungsrückstand von $1 \mathrm{ml}$ Harnextrakt aus dem Spitzröhrchen mit $2 \times 100 \mu \mathrm{l}$ Aceton $1,2 \mathrm{~cm}$ vom unteren Rand punktförmig auf eine Dünnschichtplatte von geringer Aktivität aufgetragen. Die einzelnen Urinextraktflecken sollen $3 \mathrm{~cm}$ von einander entfernt liegen. Am linken und am rechten Rand der Platte, ebenfalls $1,2 \mathrm{~cm}$ vom unteren Rand entfernt, trägt man $25 \mu l$ Steroidmischung I (je $5 \mu \mathrm{g}$ Dehydroepiandrosteron, Androsteron und Ätiocholanolon in Aceton) auf. Am rechten Rand der Platte trägt man außerdem einen Fleck einer Lösung des roten Farbstoffes Thymol-indophenol in Aceton auf. Die Dünnschichtplatte wird im System Äthylacetat/n-Hexan/ Wasser (125: $125: 0,4)$ entwickelt. Das Lösungsmittelgemisch wird vor dem Einfüllen in die Kammer kräftig geschüttelt. Da der Farbstoff etwa so schnell wandert wie Dehydroepiandrosteron, kann man mit seiner Hilfe erkennen, wie weit die Steroide gelaufen sind. In der Regel ist die am schnellsten wandernde Verbindung, Androsteron, nach 60-70 Min. in der Nähe des oberen Randes der Platte angelangt. Unter diesen Bedingungen ist Androsteron 15,6 cm, Dehydroepiandrosteron $14,0 \mathrm{~cm}$ und Ätiocholanolon $11,2 \mathrm{~cm}$ vom Startfleck entfernt. Zur Lokalisierung der Steroidflecken des Harnextraktes bläst man die Dünnschichtplatte nach der Chromatographie mit einem warmen Föhn trocken und deckt den Mittelteil der Platte möglichst dicht ab, um die Referenzsubstanzen an den Rändern mit Zimmermann-Reagenz anzusprühen. Danach erwärmt man die Randstreifen mit dem Föhn, wobei die Flecken von Androsteron, Dehydroepiandrosteron und Ätiocholanolon sichtbar werden. Eventuell muß man nochmal besprühen und exwärmen, um die Farbe besser sichtbar werden zu lassen. Man zieht 1,5 cm links und rechts von den Startflecken des Harnextraktes parallel zur Laufrichtung der Platte Striche und markiert nun die Lage der Steroide anhand dẹr angefärbten Flecken an den Rändern. Die einzelnen Rechtecke werden ausgekratzt, das Aluminiumoxid überführt man in Zentrifugenröhrchen mit Schliff. Außerdem kratzt man ein gleichgroßes Areal von einer Stelle der Platte aus, wo kein Harnextrakt gelaufen war, die aber auch nicht angesprüht wurde (Plattenleerwert). Nachdem man zu allen Röhrchen je eine Glasperle zugegeben hat, gibt man je $2 \mathrm{~m} /$ Methanol zu, schüttelt 5 Min. in der Schüttelmaschine, zentrifugiert $5 \mathrm{Min}$. bei $3000 \mathrm{U}$./Min. und pipettiert den Überstand in Zentrifugenröhrchen mit Schliff, in denen man die Eluate am Rotationsverdampfer im Vakuum eindampft. Das Aluminiumoxid wird nochmal in der gleichen Weise eluiert.

Zur Bestimmung der 11-Hydroxy- und 11-Ketosteroide trägt man in der gleichen Weise den Verdampfungsrückstand von $3 \mathrm{~m} l$ Harnextrakt ( $=6 \mathrm{~m} l$ Harn) aus den Spitzröhrchen mit $2 \times 100 \mu l$ Aceton im mittleren Teil der Platte sowie je $25 \mu$ l Steroidmischung II (mit je $5 \mu \mathrm{g}$ $11 \beta$-Hydroxyandrosteron, 11-Ketoandrosteron, $11 \beta$ Hydroxyätiocholanolon und 11-Ketoätiocholanolon in Aceton) am linken und rechten Rand einer stark aktivierten Aluminiumoxidplatte auf und entwickelt 3 Stdn. mit dem System Äthylacetat/Cyclohexan (65 : 35). Nach dieser Zeit befindet sich das am schnellsten wandernde Steroid, $11 \beta$-Hydroxyandrosteron, im oberen Viertel der Dünnschichtplatte. Nach Ansprühen der beiden Ränder mit Zimmermann-Reagenz werden die Flecken der Referenzsubstanzen sichtbar. Bei uns hatten die Steroide folgende Laufstrecken: $11 \beta$-Hydroxyandrosteron $14,5 \mathrm{~cm}, 11-$ Ketoandrosteron $11,8 \mathrm{~cm}, 11 \beta$ Hydroxyätiocholanolon $9,0 \mathrm{~cm}$ und 11-Ketoätiocholanolon 7,4 cm. Die Flecken werden in der gleichen Weise eluiert wie bei der anderen Platte.

In die Zentrifugenröhrchen mit den Verdampfungsrückständen der Eluate sowie in ein leeres Röhrchen (Reagenzienleerwert) pipettiert man $0,4 \mathrm{ml} 1$ proz. äthanol. m-Dinitrobenzollösung, schüttelt um und versetzt mit $0,3 \mathrm{ml} 8 \mathrm{~N} \mathrm{KOH}$. Nach Durchmischen läßt man die Röhrchen $25 \mathrm{Min}$. in einem Wasserbad von $25^{\circ} \mathrm{im}$ Dunkeln stehen und versetzt dann mit je $2 \mathrm{ml} 75$ proz. Äthanol (8). Alle Hauptwerte und der Plattenleerwert werden gegen den Reagenzienleerwert in $1 \mathrm{~cm}$-Küvetten im Photometer „Eppendorf" mit Filter $546 \mathrm{~nm}$ gemessen. Vor der Berechnung zieht man von den Extinktionen der Hauptwerte die Extinktion des Plattenleerwertes ab. $\mathrm{Da}$ der Plattenleerwert bei Verwendung des gleichen Adsorbens und der gleichen Lösungsmittel recht konstant ist, genügt es, wenn man ihn gelegentlich kontrolliert. Zur Bestimmung der Standardwerte pipettiert man $50 \mu l$ Standard-Lösung jedes der sieben Steroide mit je $5 \mu \mathrm{g}$ Steroid in Zentrifugenröhrchen, läßt je $1 \mathrm{ml}$ Methanol an den Innenwandungen der Röhrchen herunterfließen und verdampft das Lösungsmittel im Vakuum. Mit den Standardproben macht man in der gleichen Weise die Farbreaktion und benutzt die erhaltenen Werte zur Berechnung.

\section{Ergebnisse}

Ricbtigkeit: Einem vorher analysierten Harnextrakt wurden je $5 \mu \mathrm{g}$ der sieben Steroide zugesetzt. Die Wiederfindungen sind in Tabelle 1 angegeben.

Aluminiumoxidsäule: Es wurden $10 \mu \mathrm{g}$ jedes Steroids auf die Säule gegeben. Die Wiederfindung betrug für Dehydroepiandrosteron, 11-Ketoätiocholanolon, $11 \beta$ Hydroxyandrosteron und $11-$ Ketoandrosteron $99 \%$, für 
Ätiocholanolon, Androsteron und $11 \beta$-Hydroxyätiocholanolon $97 \%$.

Genauigkeit: Sie wurde durch eine achtfache Analyse eines Mischharnes bestimmt (Tab. 2).

Empfindlicbkeit: Sie gibt die kleinste sicher nachzuweisende Menge Substanz an. Sie beträgt für alle 17Ketosteroide je $0,2 \mu \mathrm{g} / 1 \mathrm{~m} l$ Harn.

Normaliverte: In Tabelle 3 sind die Ergebnisse der Bestimmungen an 10 männlichen und 8 weiblichen nicht bettlägerigen Normalpersonen im Alter von 16-43 Jahren zusammengefaßt.

\section{Diskussion}

Für die dünnschichtchromatographische Trennung der 17-Ketosteroide hat sich Aluminiumoxid besser bewährt als Kieselgel. Die Trennung ist schärfer und nach dem Besprühen der Aluminiumoxidplatten mit ZimmermannReagenz lassen sich die Flecken besser sichtbar machen als auf einer Kieselgelschicht. Die Schwierigkeit bei der Trennung der 11-Desoxy-17-ketosteroide liegt darin, daß Dehydroepiandrosteron und Androsteron immer recht dicht beieinander liegen. Die Durchlaufchromatographie im System Äthylacetat/n-Hexan (1:1, wassergesättigt) mit Aluminiumoxid G-Platten ergibt hin-

Tab. 1

Richtigkeit der Methode Ergebnis der Wiederfindungsversuche mit je $5 \mu \mathrm{g}$ zugesetzten Steroiden. DHA = Dehydroepiandrosteron; A = Androsteron;

\begin{tabular}{|c|c|c|c|c|c|c|c|c|c|c|c|c|c|}
\hline \multicolumn{2}{|c|}{ DHA } & \multicolumn{2}{|c|}{ A } & \multicolumn{2}{|c|}{$\mathrm{E}$} & \multicolumn{2}{|c|}{ 11-OH-A } & \multicolumn{2}{|c|}{ 11-Keto-A } & \multicolumn{2}{|c|}{ 11-OH-E } & \multicolumn{2}{|c|}{ 11-Keto-E } \\
\hline$\mu \mathrm{g}$ & $\%$ & $\mu 9$ & $\%$ & $\mu_{\mathrm{g}}^{\mathrm{g}}$ & $\%$ & $\mu \mathrm{g}$ & $\%$ & $\mu_{\mathcal{J}}^{\prime}$ & $\%$ & $\mu \mathrm{g}$ & $\%$ & $\mu \mathrm{g}$ & $\%$ \\
\hline $\begin{array}{l}4,5 \\
4,5 \\
4,3 \\
4,8 \\
4,1 \\
4,4\end{array}$ & $\begin{array}{l}90 \\
90 \\
86 \\
96 \\
82 \\
88 \\
\end{array}$ & $\begin{array}{l}4,3 \\
4,5 \\
4,6 \\
4,9 \\
4,2 \\
4,8\end{array}$ & $\begin{array}{l}86 \\
90 \\
92 \\
98 \\
84 \\
96 \\
\end{array}$ & $\begin{array}{l}4,6 \\
4,1 \\
4,8 \\
4,7 \\
4,3 \\
4,9\end{array}$ & $\begin{array}{l}92 \\
82 \\
96 \\
94 \\
86 \\
98 \\
\end{array}$ & $\begin{array}{l}4,2 \\
4,7 \\
3,8 \\
4,7 \\
4,3 \\
4,4\end{array}$ & $\begin{array}{l}84 \\
94 \\
76 \\
94 \\
86 \\
88 \\
\end{array}$ & $\begin{array}{l}4,6 \\
5,0 \\
4,6 \\
4,8 \\
4,6 \\
4,7\end{array}$ & $\begin{array}{r}92 \\
100 \\
92 \\
96 \\
92 \\
94 \\
\end{array}$ & $\begin{array}{l}5,1 \\
4,9 \\
4,8 \\
4,7 \\
4,7 \\
3,9\end{array}$ & $\begin{array}{r}102 \\
98 \\
96 \\
94 \\
94 \\
78 \\
\end{array}$ & $\begin{array}{l}4,6 \\
4,1 \\
5,0 \\
5,3 \\
4,5 \\
4,3\end{array}$ & $\begin{array}{r}92 \\
82 \\
100 \\
106 \\
90 \\
86 \\
\end{array}$ \\
\hline & $\overline{89}$ & & $\overline{91}$ & & $\overline{91}$ & & $\overline{87}$ & & $\overline{94}$ & & $\overline{93}$ & & $\overline{93}$ \\
\hline
\end{tabular}

Tab. 2

Genauigkeit der Methode Mehrfachbestimmung bei einem Mischharn

\begin{tabular}{|c|c|c|c|c|c|c|}
\hline \multicolumn{7}{|c|}{ Gehalt an $17-$ Ketosteroiden in $\mu \mathrm{g} / 10 \mathrm{ml}$ Harn } \\
\hline DHA & A & $\mathrm{E}$ & $11-\mathrm{OH}-\mathrm{A}$ & 11-Keto-A & $11-\mathrm{OH}-\mathrm{E}$ & 11-Keto-E \\
\hline $\begin{array}{l}30,0 \\
31,6 \\
32,0 \\
34,2 \\
32,0 \\
30,5 \\
30,5 \\
33,0\end{array}$ & $\begin{array}{l}61,0 \\
56,5 \\
51,5 \\
57,0 \\
53,5 \\
59,5 \\
72,5 \\
64,0\end{array}$ & $\begin{array}{l}52,0 \\
54,5 \\
52,5 \\
55,5 \\
58,5 \\
57,5 \\
63,5 \\
62,5\end{array}$ & $\begin{array}{l}2,7 \\
2,4 \\
2,2 \\
2,3 \\
2,0 \\
2,5 \\
2,3 \\
2,4\end{array}$ & $\begin{array}{l}2,3 \\
2,3 \\
2,2 \\
1,3 \\
1,4 \\
1,6 \\
2,2 \\
2,1\end{array}$ & $\begin{array}{l}4,2 \\
4,0 \\
3,3 \\
4,7 \\
4,8 \\
5,1 \\
4,7 \\
4,5\end{array}$ & $\begin{array}{l}6,0 \\
6,0 \\
5,7 \\
5,7 \\
5,6 \\
5,2 \\
5,9 \\
5,9\end{array}$ \\
\hline $\begin{array}{c}\bar{x}=31,7 \\
s= \pm 1,3 \\
( \pm 4 \%)\end{array}$ & $\begin{array}{c}59,4 \\
\pm 6,6 \\
( \pm 11 \%)\end{array}$ & $\begin{array}{c}57,0 \\
\pm 4,3 \\
( \pm 8 \%)\end{array}$ & $\begin{array}{c}2,4 \\
\pm 0,2 \\
( \pm 8 \%)\end{array}$ & $\begin{array}{c}1,9 \\
\pm 0,4 \% \\
( \pm 21 \%)\end{array}$ & $\begin{array}{c}4,4 \\
\pm 0,6 \\
( \pm 14 \%)\end{array}$ & $\begin{array}{c}5,8 \\
\pm 0,3 \\
( \pm 5 \%)\end{array}$ \\
\hline
\end{tabular}

Tab. 3

Ergebnisse der fraktionierten 17-Ketosteroidbestimmung in mg im 24-Stdn.-Harn bei gesunden Personen

\begin{tabular}{|c|c|c|c|c|c|c|c|c|}
\hline Name & Alter & DHA & A & $\mathrm{E}$ & 11-OH-A & 11-Keto-A & 11-OH-E & 11-Keto- $\dot{E}$ \\
\hline \multicolumn{9}{|l|}{ Männer } \\
\hline \multirow[t]{2}{*}{$\begin{array}{l}\text { A. B. } \\
\text { P. J. } \\
\text { E. H. } \\
\text { J. H. } \\
\text { G. V. } \\
\text { V. C. } \\
\text { H. B. } \\
\text { F. B. } \\
\text { J. F. } \\
\text { F. K. }\end{array}$} & $\begin{array}{l}21 \\
41 \\
28 \\
18 \\
35 \\
16 \\
30 \\
30 \\
19 \\
32 \\
\end{array}$ & $\begin{array}{l}2,6 \\
4,7 \\
6,9 \\
0,9 \\
3,0 \\
1,0 \\
2,0 \\
4,0 \\
2,0 \\
6,5 \\
\end{array}$ & $\begin{array}{l}3,7 \\
5,1 \\
4,7 \\
4,2 \\
2,1 \\
4,1 \\
6,5 \\
5,2 \\
4,5 \\
5,1 \\
\end{array}$ & $\begin{array}{l}5,8 \\
4,3 \\
3,2 \\
4,8 \\
2,0 \\
2,6 \\
5,6 \\
5,3 \\
3,7 \\
4,2 \\
\end{array}$ & $\begin{array}{l}0,5 \\
0,5 \\
0,5 \\
0,5 \\
0,7 \\
0,5 \\
0,6 \\
0,7 \\
0,6 \\
0,5 \\
\end{array}$ & $\begin{array}{l}0,6 \\
0,6 \\
0,4 \\
0,3 \\
1,0 \\
0,3 \\
0,3 \\
0,2 \\
0,2 \\
0,3 \\
\end{array}$ & $\begin{array}{l}1,0 \\
0,8 \\
0,8 \\
0,7 \\
1,9 \\
0,5 \\
1,1 \\
0,8 \\
0,9 \\
0,9 \\
\end{array}$ & $\begin{array}{l}0,5 \\
0,8 \\
0,9 \\
0,7 \\
0,9 \\
0,7 \\
1,2 \\
0,5 \\
0,5 \\
0,8 \\
\end{array}$ \\
\hline & $\begin{array}{r}\bar{x}= \\
s= \\
\bar{x} \pm 2 s= \\
\end{array}$ & $\begin{array}{l}3,4 \\
\pm \quad 2,1 \\
0-7,6\end{array}$ & $\begin{array}{c}4,5 \\
\pm \stackrel{1,1}{2,3-6,7}\end{array}$ & $\begin{array}{l}4,1 \\
\pm \\
1,5-6,7\end{array}$ & $\begin{array}{c}\stackrel{0,6}{0,1} \\
0,4-0,8\end{array}$ & $\begin{array}{r}0,4 \\
+0,2 \\
0-0,8\end{array}$ & $\begin{array}{c}0,9 \\
\pm 0,4 \\
0,1-1,7\end{array}$ & $\begin{array}{l}\quad 0,8 \\
\pm \stackrel{0,2}{0,4-1,2}\end{array}$ \\
\hline \multicolumn{9}{|l|}{ Frauen } \\
\hline $\begin{array}{l}\text { L. J. } \\
\text { I. W. } \\
\text { E. B. } \\
\text { B. M. } \\
\text { M. V. } \\
\text { F. D. } \\
\text { L. S. } \\
\text { E. S. }\end{array}$ & $\begin{array}{l}39 \\
34 \\
43 \\
29 \\
42 \\
41 \\
42 \\
20\end{array}$ & $\begin{array}{l}2,8 \\
0,9 \\
0,5 \\
1,4 \\
0,9 \\
3,0 \\
0,8 \\
3,5\end{array}$ & $\begin{array}{l}4,2 \\
1,5 \\
1,3 \\
3,1 \\
2,2 \\
2,8 \\
0,8 \\
1,9\end{array}$ & $\begin{array}{l}3,7 \\
2,0 \\
3,2 \\
1,9 \\
3,6 \\
3,0 \\
2,0 \\
2,8\end{array}$ & $\begin{array}{l}0,7 \\
0,3 \\
0,5 \\
0,4 \\
0,3 \\
0,4 \\
0,5 \\
0,1\end{array}$ & $\begin{array}{l}0,4 \\
0,4 \\
0,6 \\
0,3 \\
0,3 \\
0,3 \\
0,5 \\
0,2\end{array}$ & $\begin{array}{l}0,8 \\
0,7 \\
1,0 \\
0,9 \\
1,1 \\
0,6 \\
1,0 \\
0,4\end{array}$ & $\begin{array}{l}0,7 \\
0,3 \\
0,4 \\
0,7 \\
0,6 \\
0,4 \\
0,4 \\
0,7\end{array}$ \\
\hline$\cdot$ & $\begin{array}{r}\bar{x}= \\
s= \\
\bar{x} \pm 2 s=\end{array}$ & $\begin{array}{rl} & 1,7 \\
\pm & 1,2 \\
0 & 4,1\end{array}$ & $\begin{array}{r}2,2 \\
+1,1 \\
0-4,4\end{array}$ & $\begin{array}{l}2,8 \\
\pm \stackrel{0,7}{1,4-4,2}\end{array}$ & $\begin{array}{r}0,4 \\
\pm 0,2 \\
0-0,8\end{array}$ & $\begin{array}{c}0,4 \\
\pm 0,2 \\
0-0,8\end{array}$ & $\begin{array}{c}0,8 \\
+0,2 \\
0,4-1,2\end{array}$ & $\begin{array}{c}0,5 \\
\pm 0,2 \\
0,1-0,9 .\end{array}$ \\
\hline
\end{tabular}


gegen eine gute Trennung von Dehydrocpiandrosteron, Androsteron und Ätiocholanolon. Der Vorteil der Durchlaufchromatographic liegt darin, daß man die Laufzeit nach Belieben verlängern und die Trennung damit verbessern kann. Nach 60-70 Min. ist die schnellste Substanz (Androsteron) in der Nähe der Front. Die 11-oxygenierten 17-Ketosteroide haben eine viel geringere Laufgeschwindigkeit als Ätiocholanolon. Sie sind somit auf dieser Platte in diesem System nicht getrennt. $\mathrm{Zu}$ ihrer Trennung verwenden wir Aluminiumoxid G-Platten mit höherer Aktivität, die wir nach dem Durchlaufverfahren im System Äthylacetat/Cyclohexan $(65: 35)$ entwickeln. Nach einer Laufzeit von 3 Stdn. sind die 11-oxygenierten Verbindungen gut getrennt, während Androsteron, Dehydroepiandrosteron und Ätiocholanolon bereits bis zur Front durchgelaufen sind. Die für die Durchlaufchromatographie verwendeten Kammern lassen sich ohne große Kosten aus den üblichen Chromatographiegefäßen herstellen. Wir haben mit dieser Anordnung immer gute Trennungen erzielt.

Wenn der Harnextrakt stark gefärbt ist, was häufig bei pathologisch veränderten Harnen vorkommt, wird zu viel Verunreinigung mit auf die Dünnschichtplatte aufgetragen. Vom Startfleck zieht sich dann ein brauner „Schwanz" bis in die Mittc der Platte und stört besonders die Bestimmung der 11-oxygenierten Steroide. Man erhält dann $z u$ hohe Werte. Durch eine einfache Filtration durch eine kleine Aluminiumoxidsäule läßt sich der Extrakt gut und leicht vorreinigen.
Dabei werden $97-99 \%$ der vorgelegten Steroide wiedergefunden.

Die Kombination von enzymatischer Hydrolyse und Solvolyse führt zu einer praktisch vollständigen Spaltung der Konjugate (7). In mehreren zuvor enzymatisch und solvolytisch hydrolysierten Harnen, die mit Äthylacetat extrahiert waren, fanden sich nach anschließender "saurer Hydrolyse" und Extraktion mit Äther nur noch etwa $1 \%$ der ursprünglich durch enzymatische Hydrolyse gefundenen Gesamt-17-Ketosteroide.

Bei allen untersuchten Harnen bestimmten wir gleichzeitig die Gesamt-17-Ketosteroide nach der Methode von ZimmermanN in der Modifikation von WaLter (9). Bei 30 Harnen betrug die Summe der sieben chromatographisch getrennten Steroide durchschnittlich $84 \%$ der sog. Gesamt-17-Ketosteroide. Die Bestimmung der Gesamt-17-Ketosteroide nach ZIMMERMANN ist bekanntlich nicht ganz spezifisch und gibt stets zu hohe Werte. Die Summe der dünnschichtchromatographisch getrennten Steroide dürfte also dem ,wahren" 17-Ketosteroidgehalt etwa entsprechen. Die Methode trennt die wichtigsten 17-Ketosteroide, nämlich Dehydroepiandrosteron, Androsteron, Ätiocholanolon, $11 \beta$-Hydroxyandrosteron, 11-Ketoandrosteron, $11 \beta$-Hydroxyätiocholanolon und 11-Ketoätiocholanolon schnell, sauber und spezifisch. Sowohl Empfindlichkeit als auch Richtigkeit und Genauigkeit sind gut. Deshalb ist die Methode für die routinemäßige Bestimmung dieser Verbindungen geeignet.

\title{
Literatur
}

1. Reisert, P. M. und D. Schumacher, Experientia 19, 84 (1963). - 2. Chang Shen, N. H., F. E. Francis und R. A. Kinsella, J. Laborat. Clin. Med., S. Louis 60, 1017 (1962). - 3. StiRKA, L., J. Sulcovi, J. Riedlovi und O. Adamec, Clin. chim. Acta (Amsterdam) 9, 168 (1964). - 4. Hamman, B. L. und M. M. Martin, J. Clin. Endocr., Springfield 24, 1195 (1964). - 5. Suliarovici, S., B. Lunenfeld und M. C. Shelesnyak, Acta endocr.,
K'hvn. 51, 447 (1966). - 6. Starnes, W. R., A. H. Rhodes und R. H. Lindsay, J. Clin. Endocr., Springfield 26, 1245 (1966). 7. Baulieu, E. E. und M. F. Jayle, Analyse des Steroides Hormonaux, Tome I, S. 73, Masson et Cie., Paris (1961). - 8. Drekter, I. J., G. R. Scism, S. Stern, S. Pearson und T. H. McGavaCK, J. Clin. Endocr., Springfield 12, 55 (1952). - 9. WaLter, K., Klin. Wschr. 30, 474 (1952).

\section{Erfahrungen über die optimale Darstellung und Diagnostik der Gc-Typen ${ }^{1}$ )}

\author{
Von R. HII.GÉRMANN \\ Aus dem Institut für gerichtliche Medizin der Universität Marburg (Direktor: Prof. Dr. F. Scbleyer)
}

(Eingegangen am 20. Mai 1967)

\begin{abstract}
Die Zuverlässigkeit der Variante der immunoelektrophoretischen Mikromethode auf Glasplatten zur Gc-Klassifizierung wurde geprüft. Die Vorteile dieses Verfahrens und seine besondere Eignung in der Routinediagnostik werden gegenüber der Objektträgermethode hervorgehoben. Es wird gezeigt, daß sich nicht nur auf Grund zahlreicher verfahrensmäßiger Modifikationen, sondern auch bei scheinbar gleichen Versuchsbedingungen unterschiedliche Auftrennungszeiten ergeben. Daher wird empfohlen, die Laufstrecke der Proteine als Richtgröße festzulegen, um reproduzierbare Ergebnisse zu erhalten. Der Calciumlactatpuffer erlaubt eine zuverlässigere Diagnostik als der Michaelispuffer.

The rcliability of the modified immuno-electrophoretic micromethod on glass plates was tested. The advantages of this procedure over the slide method, and its special suitability for routine diagnosis are shown. Variations in the separation times, which are not entircly duc to the many modifications of the method, are observed, even under apparently identical test conditions. It is therefore recommended that the proteins are always allowed to migrate the same distance in order to obtain reproducible results. Calcium lactate buffer is morc reliable than Michaelis buffer for diagnosis.
\end{abstract}

1) Auszugsweise vorgetragen auf der 45. Tagung der Gesellschaft für gerichtliche Medizin, Freiburg 1966. 